\section{Intertest Scatter}

Sandra Banks

Department of Psychiatry, Allegheny General Hospital, Pittsburgh, PA, USA

\section{Synonyms}

Intertest variability

\section{Definition}

Intertest scatter is a measure of the variability of standardized scores on subtests within a larger test or on separate tests within a larger battery of tests. High intertest variability is depicted by a wide array of scores when the scores of more than one test are entered into a scatterplot. Low intertest variability is depicted by a minimal scattering of scores when the scores of more than one test are entered into a scatterplot. The degree of scatter needed to be considered abnormal is a function of the correlation among the tests and the reliability of the individual scores. Scatter in tests that do not correlate highly may not reflect pathology. Scatter among scores with low reliability may not reflect pathology.

Specific patterns of scores on tests may assist with diagnosis. For example, when an individual's scores are consistently intact across the indices of the Wechsler Intelligence Scale for Children Fifth Edition (low scatter) with the exception of variable performance on the Working Memory Index (high scatter when compared with other subtests), this may indicate attention difficulties. Within a battery of tests, if an individual demonstrates relatively intact performance in the majority of cognitive domains but has weaknesses on memory and language tasks, one might consider brain injury or dementia, depending on the patient's presenting concerns and history.

\section{Cross-References}

Test Interpretations 